\title{
A New Type of Measurement System for Gasoline Engine Control Parameters
}

\author{
Tiezao Yang ${ }^{1, ~ a, ~ H a i b o ~ X u e ~}{ }^{2, b}$, Xinyang Wang ${ }^{1, b}$, Changsheng Wang ${ }^{1, c}$ \\ ${ }^{1}$ College of Vehicle and Motive Power Engineering, Henan University of Science and Technology, \\ Luoyang, Henan, 471003, China \\ ${ }^{2}$ Henan Diesel Engine Industry Co., Ltd, Luoyang, Henan, 471003, China \\ aytz8341@126.com, bxuehaibo11@qq.com, cwangxiaoouou@163.com
}

\begin{abstract}
Keywords: Gasoline Engine, the Theoretical Parameters, the Practical Parameters, the Control Parameters.

Abstract: It is important whether the theoretical control parameters of the gasoline engine, such as ignition advance angle, the fuel injection advance angle, the closing time, the injection pulse width and so on, are in accord with the actual control parameters, because they relate to the role on a new product development of the gasoline engine. With the conventional experimental method, the experimental error and the difficulty during the experimentation have existed. This paper provides a new method for the experiment of gasoline engine. The core ideas of the new method, which can solve the problems of large experimental error and the difficulty during the experimentation, is to extract the theoretical parameters and measure the actual parameters in the same working conditions.
\end{abstract}

\section{Introduction}

The control parameters of the gasoline engine, such as ignition advance angle, the fuel injection advance angle, the closing time, the injection pulse width and so on, are measured by conventional experimental methods as follows ${ }^{[1]}$ : Firstly, the change rules of operating conditions in the experimental scheme is discovered, according to the change rules in MAP diagram to get the theoretical control parameters. Secondly, when the experiment is performed according to the change rules, the actual parameters will be abstained. At last, computing similarity between the theoretical parameters and actual parameters ${ }^{[2]}$. Obviously, the method is feasible to get the rules of theoretical parameters and actual parameters, but there are some disadvantages ${ }^{[3]}$. On the one hand, the operating point of theoretical data is accurate, and the operating point of the actual parameters need to debug the engine, confirm it, which is in line with the theoretical parameters of the operating point, but the operating conditions of the two are difficult to maintain the same, both on the conditions of speed and load. Because of the different working conditions, there are obvious differences between theoretical parameters and practical parameters. On the other hand, because of the experiment based on strict working conditions, which are given by adjusting the actual parameters of gasoline engine, it is time-consuming and very difficult to experiment. The speed fluctuation is more prominent than other parameters for gasoline engine ${ }^{[4,5]}$. This paper puts forward a new solution to solve these two problems. The flowcharts provides some clues to solve the problems is shown in Fig. 1.

\section{Experimental program}

Fig. 1 shows that the experimental scheme is divided into two ways to execute the processing of data extraction and data acquisition at the same time.

The first way: execute the processing of working condition parameter and theoretical parameters extraction.
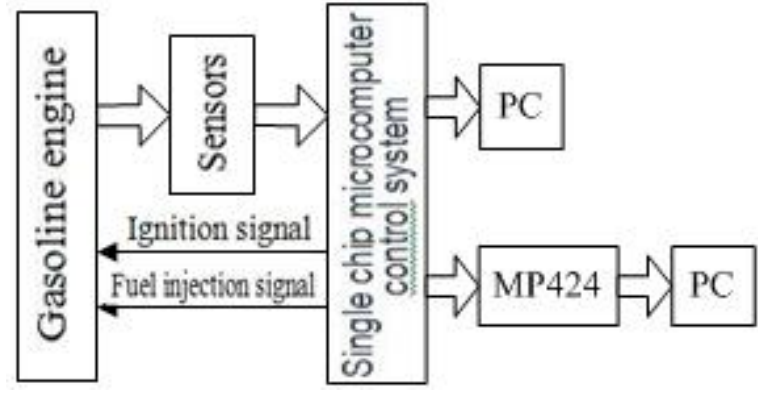

The first way

Fig. 1. Experimental scheme 
Working condition parameter: Such as speed, throttle position, engine temperature, inlet temperature and battery voltage. They are measured by the microcontroller through the sensors, and then stored in the microcontroller for the use of the software. These parameters, which can be extracted from the shared memory and serial communication, are sent to PC and displayed by the software ${ }^{[6]}$.

Theoretical parameters: The theoretical parameters is in the microcontroller memory MAP diagram on the control parameters, such as the various conditions of the ignition advance angle, fuel injection advance angle, closing time and fuel injection pulse width and other parameters. These theoretical parameters, which are extracted from MAP diagram in the microcontroller memory, are sent to the PC by shared

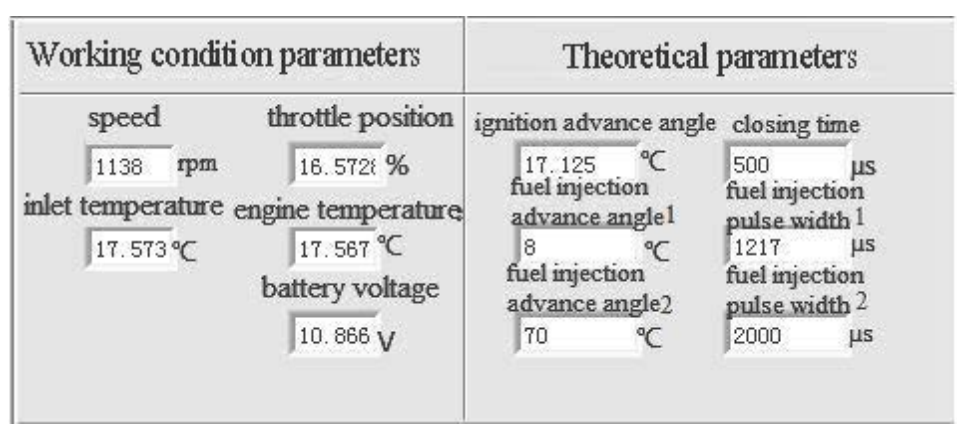

Fig. 2. Working condition parameters and theoretical parameters of a certain working condition memory and serial communication, and displayed by the software.

Fig. 2 shows that the working condition parameter and theoretical parameters of a certain working condition are extracted. The left side of the diagram shows a working condition parameter, and the right side shows a theoretical parameter. It can be seen that the experimental points are working conditions, and the theoretical parameters are from the diagram ${ }^{[7]}$.
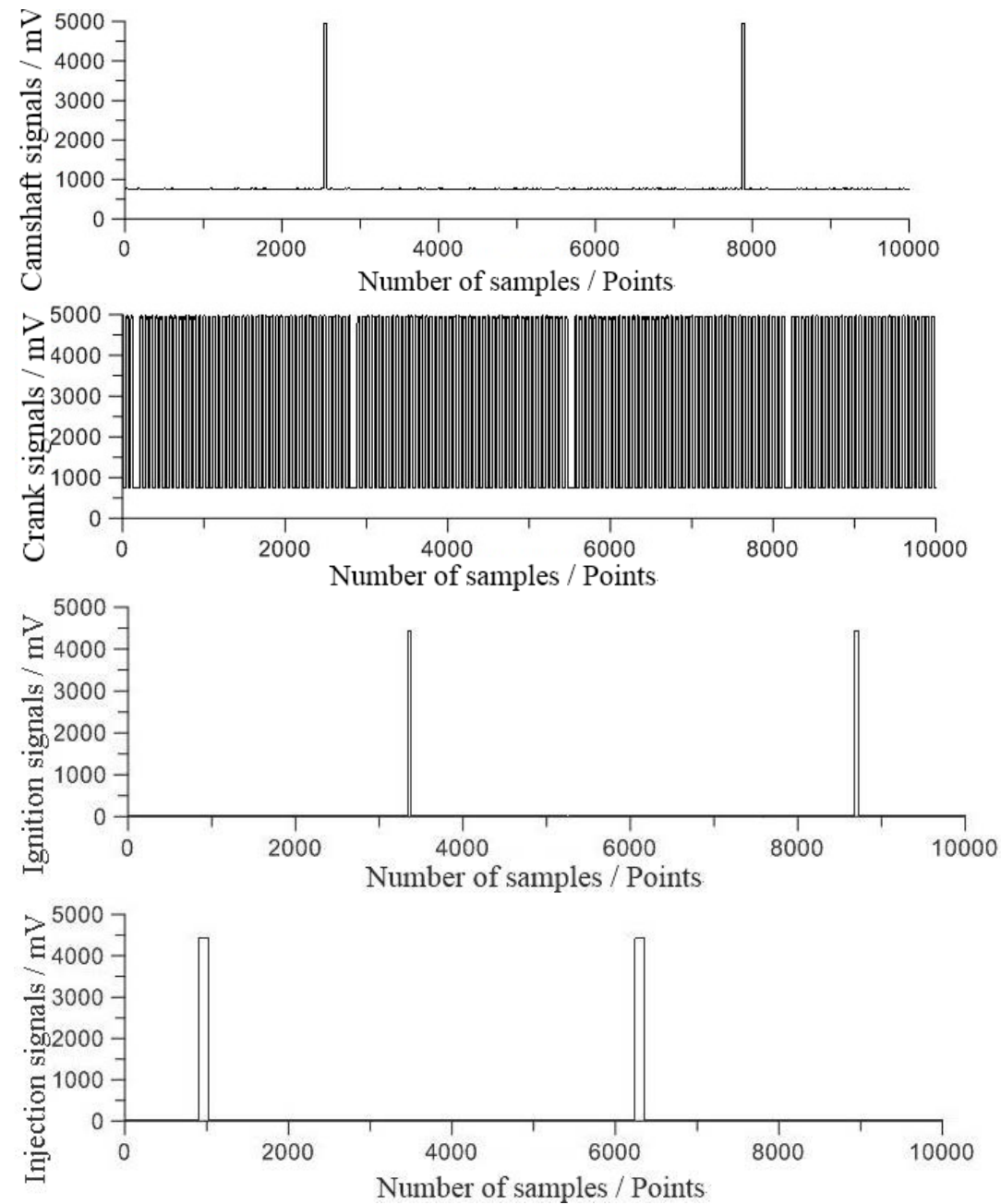

Fig. 3. MP424 acquisition of 4 signals 
The second way: Actual parameters measurement.

The actual parameters are the control parameters measured in the actual engine operation. In the actual parameters, the MP424 data acquisition card acquires the signal first from the data acquisition system. The signal include camshaft and crankshaft signal, ignition signal and fuel injection signal. Then the software of LabVIEW calculates the actual control parameters, such as ignition advance angle, fuel injection advance angle, closing time and injection pulse width ${ }^{[8]}$. A MP424 data acquisition card collects the signal of a certain working condition of the cam signal, crankshaft signal, ignition signal and fuel injection signal, as are shown in Fig. 3. Then the signal can be calculated in a certain condition of the actual ignition advance angle, fuel injection

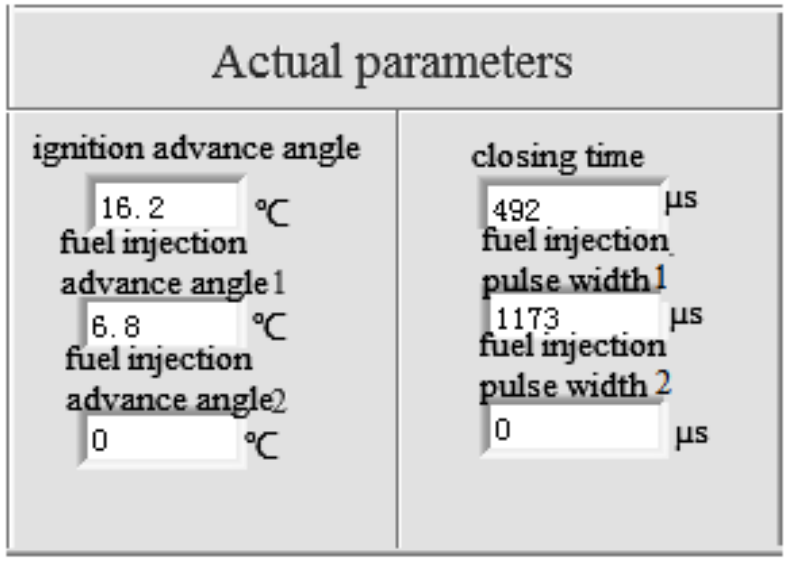

Fig. 4. The actual parameters of a certain working condition advance angle, closing time and fuel injection pulse width, as are shown in Fig. 4.

According to the scheme, the theory and the actual parameters of the acquisition and processing can be performed at the same time. If there is a difference between theoretical parameters and practical parameters, the error can reach the minimum. Besides, it is not necessary to adjust the engine to a certain operating condition, the engine only need be deliberately adjusted to a certain working condition. The results are consistent with the results of the two cases, and the error of the theory and the actual parameters can reach the minimum.

\section{Measurement process}

It is important to be clear what the frequency division signal is. In the engine, the ignition advance angle and fuel injection advance angle are 1 degrees crank angle for the unit, and each crankshaft signal is 8 degrees crank angle. In order to solve this problem, the crankshaft signal 8 is divided into frequency 1 to 1 degree angle, and the frequency division signal is a signal after the crankshaft signal 8 is divided.

This program is compiled by LabVIEW software. After the program starts to read the data file, the first test of the camshaft signal. Because an engine cycle has only one camshaft signal, the camshaft signal marks the beginning of a cycle.

When the crankshaft signal, ignition signal, fuel injection signal and frequency division signal are aligned, LabVIEW processes the signal by time-shared processing, and calculates ignition advance angle, fuel injection advance angle, closing time and injection pulse width. Finally, these parameters are extracted and displayed, as are shown in Fig. 5.

The process of closing time and fuel injection pulse width is relatively simple, and the ignition advance angle is similar to that of the fuel injection advance angle. There is the signal processing as follows, with the signal of ignition advance angle for an example.

A counter for frequency division signal is used to record the angle of the crankshaft, and the counter starts to record the crankshaft turning point, the counter begins recording timing of ignition signal falling edge, and ends at the signal of TDC. It is obvious to recognize the ignition signal falling edge, and the TDC position is in position of a crankshaft 10 rising edge of the pulse, which is behind the camshaft signal and the first missing teeth. Counter begins counting at the ignition signal falling edge, and stops counting at the TDC position. The entire counter records the number of one signal as the ignition advance angle in degrees. As are shown in Fig. 5. 


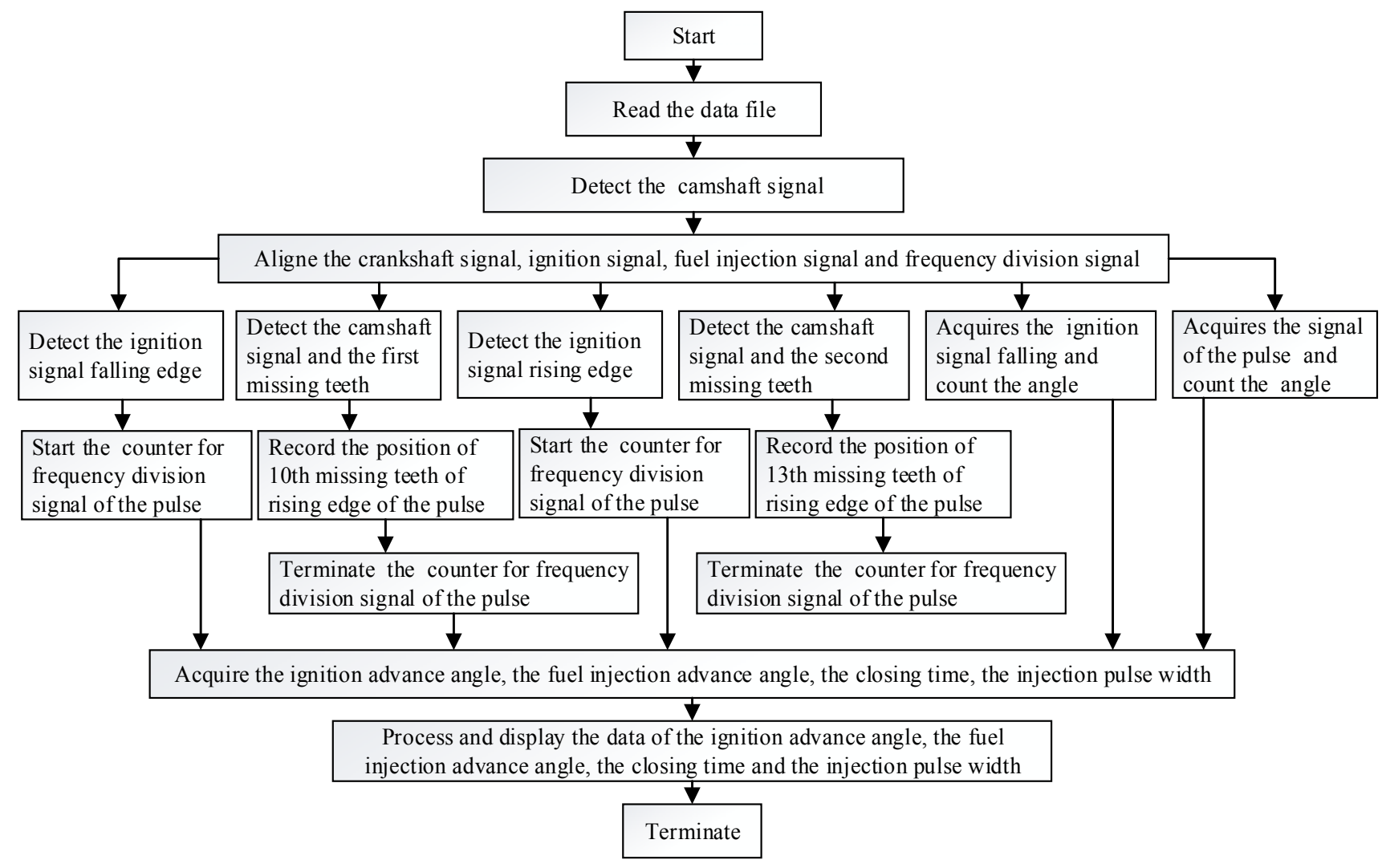

Fig. 5. The signal processing with the signal of ignition advance angle for an example

\section{Experimental Results}

The test was based on a CG125 motorcycle gasoline engine after refit ${ }^{[9,10]}$.

The electronic control engine runs with no load. There are eight test points selected following the rotational speed from low to high. Each working condition in the experiment, which does not need to strictly correspond to a certain working condition, is just found out only once. Therefore, the experiment is very convenient. The theoretical and actual parameters are measured simultaneously after the working condition is determined. The time of measurement is only about 0.01 seconds. Because the experiment time is very short, it can be considered that the two kinds of parameters are in the same working conditions. As the experiment is executed strictly in the same condition, it can eliminate the errors caused by different experimental conditions.

Because of the large amount of the experimental data, we will take the ignition advance angle as an example to analyze. The experimental data is shown in Table 1 . The parameters are analyzed and processed after the theoretical parameters extracted and the actual parameters measured. The relationship between the theoretical value and the actual

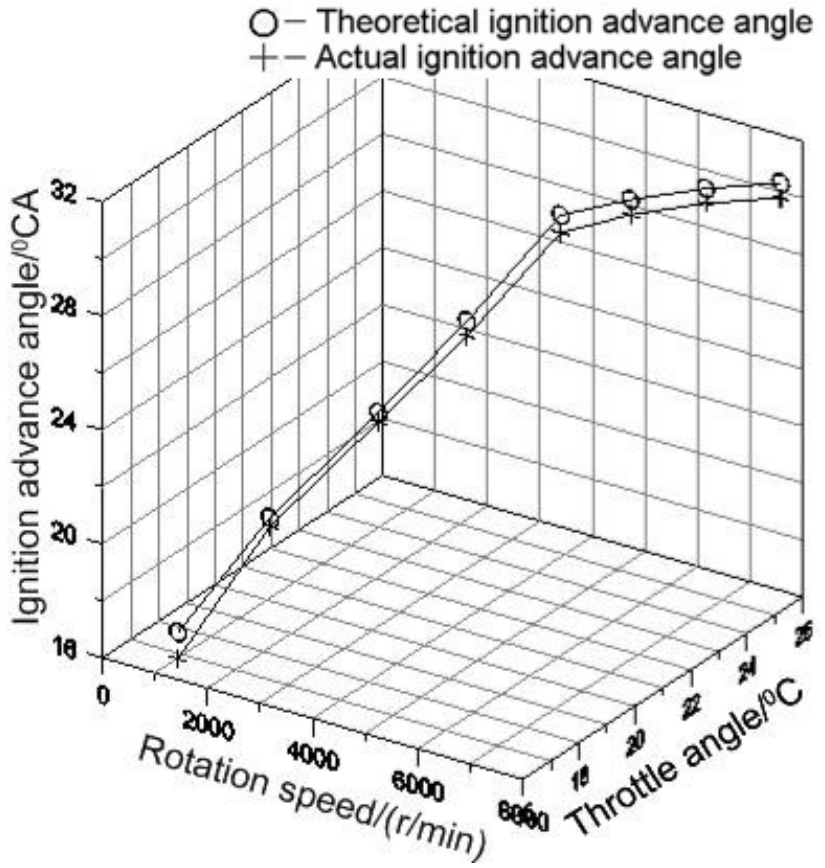

Fig. 6. Comparison between theoretical values and actual values of ignition advance angle value of the ignition advance angle with the different operating conditions is shown in Fig. 6. 
Table 1. Theoretical and actual ignition advance angle

\begin{tabular}{ccccccccc}
\hline Rotational speed $(\mathrm{r} / \mathrm{min})$ & 1138 & 1978 & 3073 & 3946 & 5050 & 6009 & 6945 & 7937 \\
\hline Throttle angle $\left(^{\circ}\right)$ & 16.57 & 18.28 & 20.09 & 21.62 & 22.89 & 23.58 & 24.52 & 25.33 \\
Theoretical ignition advance angle $\left(^{\circ}\right)$ & 17.13 & 20.51 & 23.62 & 26.33 & 29.82 & 30.43 & 30.72 & 30.93 \\
Actual ignition advance angle $\left(^{\circ}\right)$ & 16.2 & 20.2 & 23.3 & 25.8 & 29.2 & 29.9 & 30.2 & 30.4 \\
\hline
\end{tabular}

\section{Experimental Analysis}

The experimental process suggests that the experiment of each working point is probably determined near a certain working condition. For example, if we need to measure with a rotate speed $3000 \mathrm{rpm}$, we should adjust the engine rotate speed to near $3000 \mathrm{rpm}$ (such as $3073 \mathrm{rpm}$ ). It is very convenient and well adaptive throughout the whole experiment. It can be completed in a very short time. It is different from the original experiment, which needs to find the theoretical parameters and operating point parameters from the MAP diagram first, and then confirm the operating conditions and conditions in MAP diagram under the same conditions, and finally measure the actual parameters by the experiment on the engine after. Because every experiment operating point and the ideal operating point should be as consistent as possible, it is difficult and slow.

We will discuss the accuracy of it as follows. It can be seen that the theoretical value of the ignition advance angle is almost as same as the actual value from the experimental results as shown in Table 1. We will take the maximum error in $7937 \mathrm{rpm}$ for an example: The maximum difference is 0.53 degree which is relatively small.

There are two main reasons for the high accuracy: Firstly, the two conditions are in strict accordance with each other, as has already been observed. Secondly, the control scheme is based on the digital control, and the structure is reasonable. The rationality of the control scheme is discussed as follows.

There is the control schematic diagram for the ignition advance angle as shown in Fig. 7. There is the crankshaft signal which is a row of signal in the Fig. above, and each crankshaft signal is the same as 8 degrees crank angle. The ignition timing is the falling edge of the ignition signal which is a signal in the Fig. below.

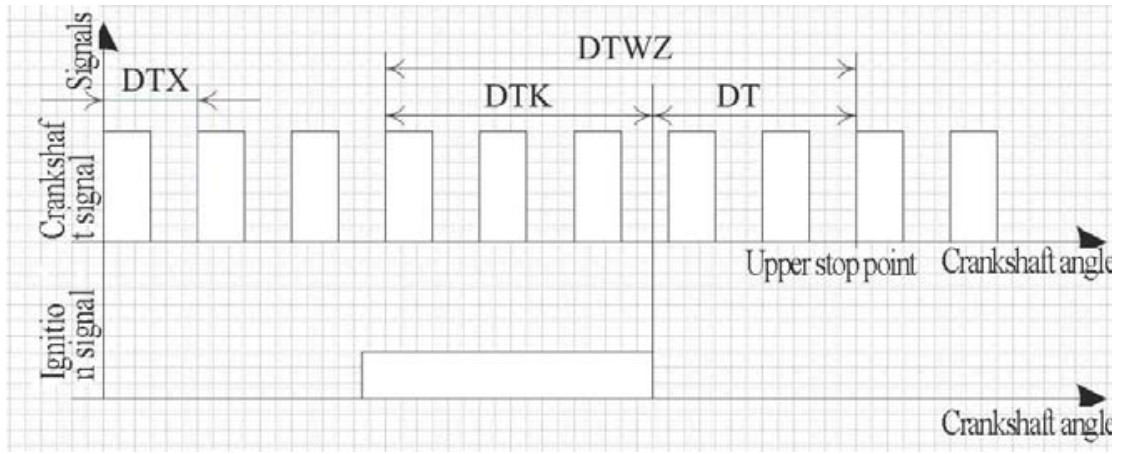

Fig. 7. Control schematic diagram for the ignition Advance angle as shown

Several parameters are stated as follows:

DTWZ: the position angle of ignition advance angle, which is a fixed value, about 40 degrees;

DT: the ignition advance angle, which is the angle of the crankshaft turning between the ignition positions to the upper stop point;

DTK: the control angle of ignition advance angle;

DTX: the count value of crankshaft signal one tooth angle.

The count value of DTX is measured by a SCM (single chip microcomputer). A DTX angle is 8 degrees, and it is clear that 1 degree angle of the crankshaft is DTX/8. The count value of DTK (the control angle of the ignition advance angle) can be calculated as follows: (DTWZDT) $\times$ DTX $/ 8$.

Obviously, when the microcontroller is executed to the baseline position of the first 40 degrees, and starts counting (DT - DTWZ) $\times$ DTX/8, after the completion of the counting is to start the ignition, and the ignition advance angle is the ignition time to the upper end of the crankshaft angle. 
From the control method of view, the control scheme is based on the digital control, so the error is small.

\section{Conclusion}

1. The accuracy of the experiment. Because the conditions of the theoretical parameters and the actual parameters are the same, the influence of the experimental results is minimal. In addition, the control method of the digital control makes the accuracy of the experiment higher.

2. The convenience of the experiment. The experiment just needs to choose a near operating point, and avoid troubles made by choosing a specific working point.

\section{References}

[1] Karl G, Rew L, Sam A, et al. The Effect of Advanced Combustion Control Features on the Performance of a Highly Downsized Gasoline Engine, Beijing, China, 2012[C].

[2] Yang T, Qian C, Wang C, et al., Hohhot, China, 2012[C]. Trans Tech Publications, 2012.

[3] Madan K, Tielong S. Estimation and feedback control of air-fuel ratio for gasoline engines[J]. Control Theory and Technology, 2015(02):151-159.

[4] Ting W X L C, Changchun U O T C, Chinay X F Z W, et al. Research in Fuel Injection Revision Control of Gasoline Engine based on the Electronic Throttle during Transient Condition, Jilin, China, 2010[C].

[5] Kano H, Fujioka H, Egerstedt M, et al., Prague, Czech republic, 2005[C]. IFAC Secretariat, 2005.

[6] Sanyal P, Shen C N. Bayes' decision rule for rapid detection and adaptive estimation scheme with space applications[J]. Automatic Control, IEEE Transactions on, 1974,19(3):228-231.

[7] Çağatay Bayindir K, Gözüküçük M A, Teke A. A comprehensive overview of hybrid electric vehicle: Powertrain configurations, powertrain control techniques and electronic control units[J]. Energy Conversion and Management, 2011,52(2):1305-1313.

[8] Wang L, Tan Y, Cui X, et al. The Application of LabVIEW in Data Acquisition System of Solar Absorption Refrigerator[J]. Energy Procedia, 2012,16, Part C(0):1496-1502.

[9] Tiezao Yang,Changsheng Wang,et al. High-speed Data Acquisition System Based on MP424 Acquisition Card[J]. Advanced Materials Research Vols.805-806(2013)pp1800-1804.

[10] Tiezao Yang, Changjun-Qian,et al. Design of the Calibration PC Software for Motorcycle Electronically Controlled Engine System based on LabVIEW[J] . Advanced Materials Research Vols.516-517(2012)pp1599-1603. 